\title{
A parallel breakout box for I/O monitoring
}

\author{
D. GOODMAN and R. MASKELL \\ Simon Fraser University, Burnaby, British Columbia, Canada
}

The construction and use of a parallel breakout box for input/output (I/O) monitoring is discussed. A standard chip for I/O is used along with circuitry, light-emitting diodes, and switches to allow researchers to simulate the running of computer-controlled experiments.

Interfacing a microcomputer to experimental apparatus is often done through a parallel port. The ease of interfacing is due largely to the use of programmable largescale integrated circuit (LSI) interface chips, which can handle different applications, and to the fact that changes and corrections can be made in software rather than by rewiring. Two of the more popular LSI interface chips that can be used with the 6502 microprocessor are the 6821 peripheral interface adapter (PIA, available from California Computer Systems, 250 Caribbean Dr., Sunnyvale, CA 94086) and the 6522 versatile interface adapter (VIA, available from J. Bell Engineering, P.O. Box 338, Redwood City, CA 94064). Here we discuss the construction and use of a parallel interface breakout box designed

The authors' mailing address is: Department of Kinesiology, Simon Fraser University, Burnaby. B.C., Canada V5A 1 S6.

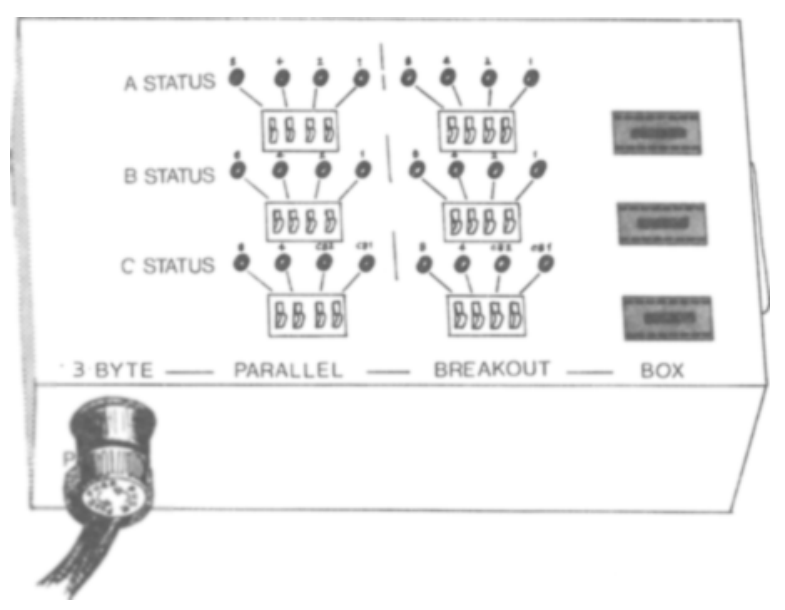

Figure 1. A diagram of the parallel breakout box.

Table 1

Parts List

\begin{tabular}{clr}
\hline Number & \multicolumn{1}{c}{ Part } & $\begin{array}{c}\text { Approximate } \\
\text { Cost }\end{array}$ \\
\hline 4 & 4049 CMOS hex inverters & $\$ 2.00$ \\
6 & Grayhill toggle dip switches No. 76STCOA & 12.00 \\
24 & Red LED & 3.00 \\
24 & $1 \mathrm{k \Omega} .25 \mathrm{~W}$ & .25 \\
1 & Hammond box 1444-10 & 2.00 \\
1 & 7805 5-V regulator TO-220 package & 1.00 \\
1 & $500-\mu$ 25-V electrolytic capacitor & .50 \\
1 & 1- $\mu$ 10-V tantalum capacitor & .75 \\
1 & .25-A fuse & .20 \\
1 & Fuse block panel mount & .50 \\
1 & DB-25 connector (male) & 2.50 \\
1 & 3-conductor power cable with molded plug & 2.00 \\
1 & Strain relief for power cable & .10 \\
1 & Vero wirewrap board (3.75 $\times 5.75$ in.) & .50 \\
6 & Standoffs .5 in. with mounting hardware & .10 \\
Many & T44DP wirewrap posts to mount leads, resistors, etc. & \\
7 & 16 pin wirewrap dip sockets & 2.00 \\
6 & 14 pin wirewrap dip sockets & 1.50 \\
1 & 1-A 50-V bridge rectifier & 1.50 \\
1 & Hammond transformer model 166G6 & 6.00 \\
Total & & $\$ 38.40$ \\
\hline
\end{tabular}




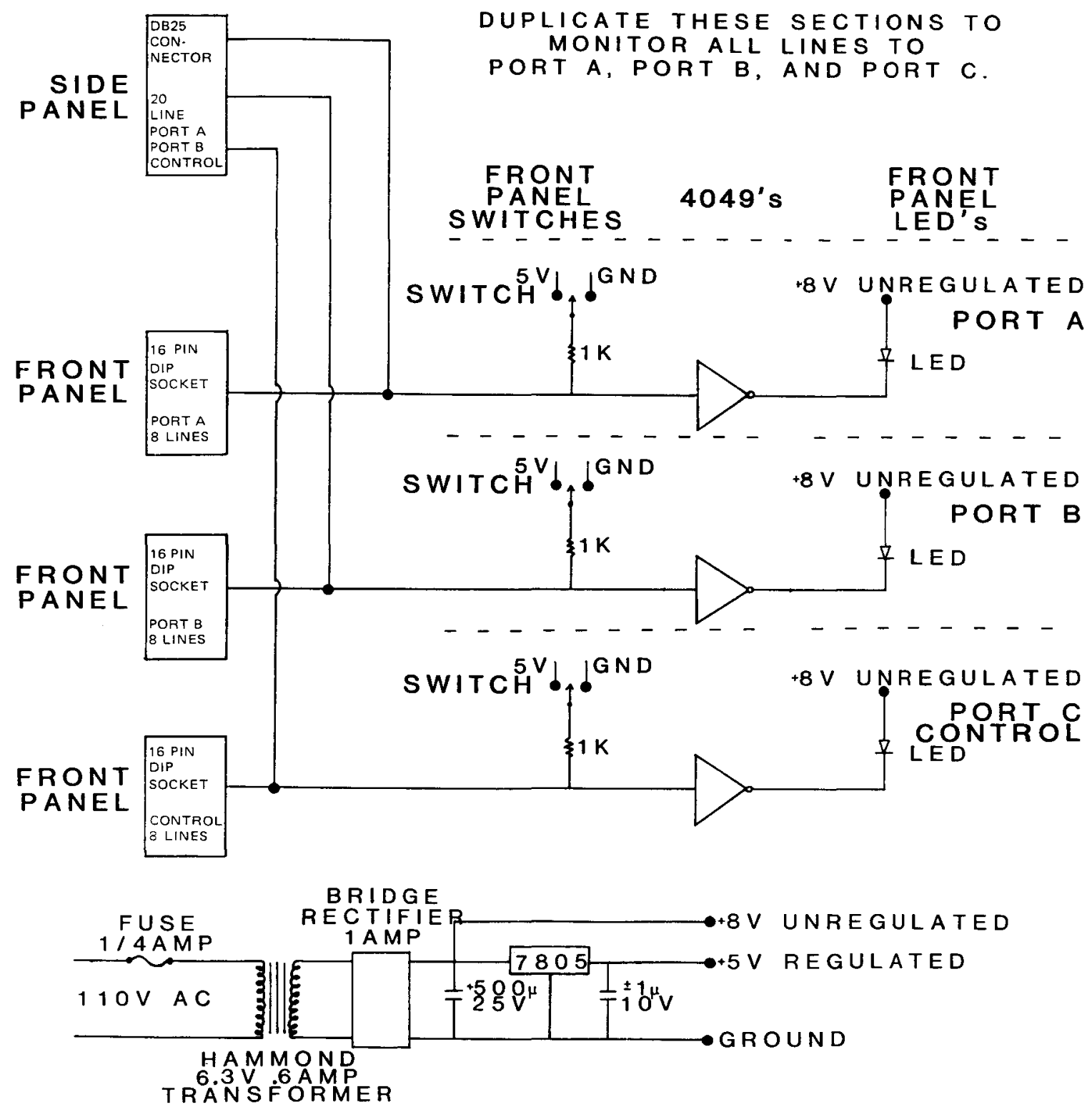

Figure 2. Schematics for construction of the parallel breakout box.

specifically for use with the CCS 7720 . The box is readily adaptable for use with the VIA parallel interface.

The parallel breakout box is a simple input/output (I/O) monitoring device to enable Apple II users to quickly determine the PIA card setup. More importantly, it allows a researcher to simulate running an experiment with or without the external devices attached. This allows quick determination of software applicability and the detection of equipment malfunction. Inclusion of a breakout box at each computer workstation of different apparatus allows quick and easy attachment to the computer.

The design approach is based on the simplicity of the PIA itself (Leventhal, 1979). The device contains two 8bit I/O ports and four individual control lines. Thus, the breakout box should be able to monitor a minimum of 20 TTL I/O lines. We chose to monitor the output status by use of light-emitting diodes (LEDs). With bit switches, any bit pattern ( 16 bits maximum) can be input to the PIA by the breakout box. The four control lines can also be controlled by the bit switches. Connections are made via the standard DB25 pin connector and 16 pin DIPS. The breakout box is illustrated in Figure 1 .

\section{CONSTRUCTION OF THE BREAKOUT BOX}

The electronics (Figure 2) are simply replicated for each of the 20 lines coming from the PIA. All that is needed is a hex inverter (4049), l-k $\Omega$ resistor, switch, and LED 
for each line. The electronics are mounted directly on a wirewrap Vero board that is then enclosed in a Hammond 1444-10 aluminum case. By the use of standoffs, the board is mounted so that all the LEDs and Grayhill toggle DIP switches protrude through the front panel. To make the box versatile, a DB25 connector is also mounted on the side panel of the case. Access to the PIA I/O lines is also possible via 16 pin DIP sockets on the front panel. Power for the interface comes from the host computer or suitable supply. A parts list is presented in Table 1.

\section{USE OF THE PARALLEL BREAKOUT BOX}

Software support for the breakout box is listed in the Appendix. One can either determine a bit pattern to send out to the box or read the information from the bit pattern of switches. Thus, prior to running an experiment, the apparatus can be tested by simply connecting to the breakout box. Similarly, the software to control experimental apparatus can be run without the actual apparatus. In this case, the breakout box simply simulates the apparatus.

\section{REFERENCE}

LeVenthaL, L. A. (1979). Assembly language programming. Berkeley, CA: Osborne/McGraw-Hill

\section{APPENDIX}

Software Support for the Breakout Box

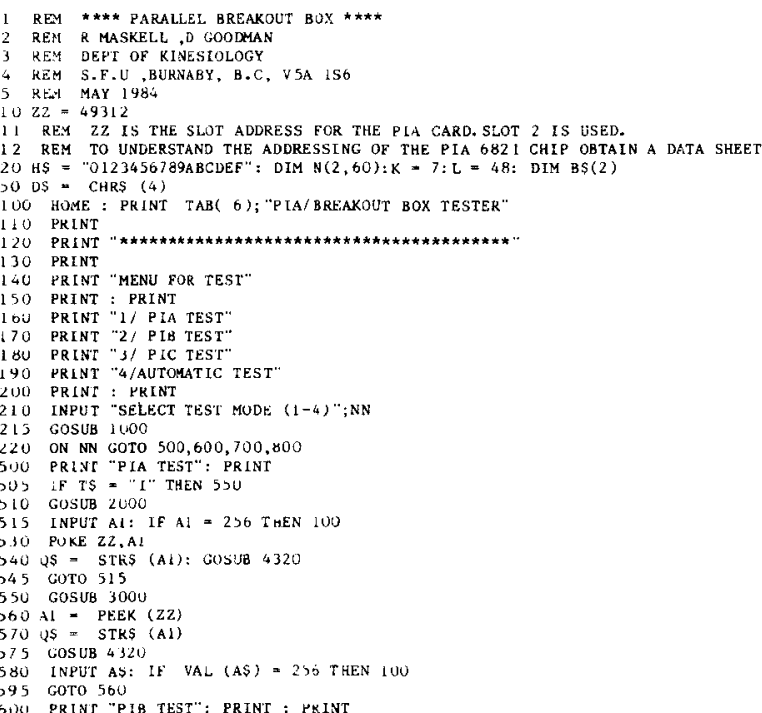

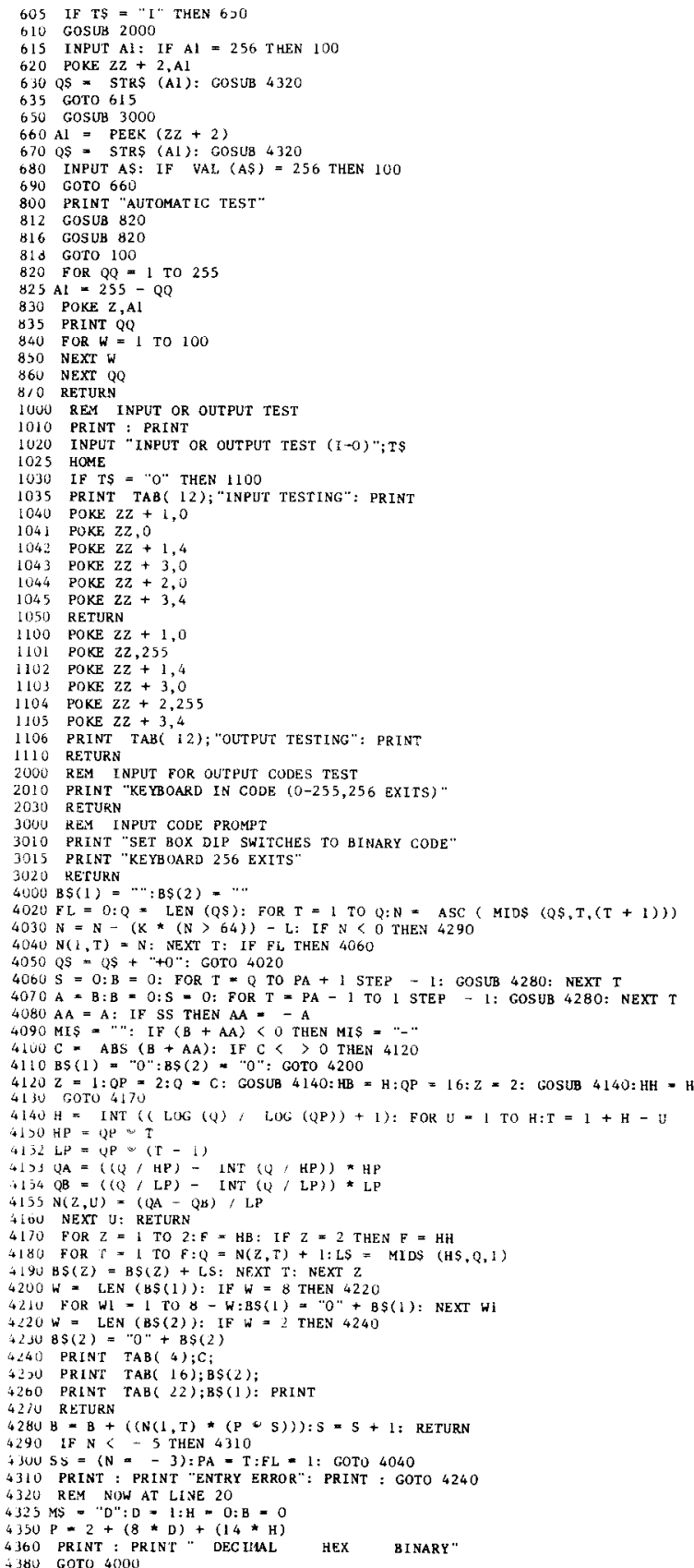

(Manuscript received December 1, 1984; revision accepted for publication March 6, 1985.) 